\title{
La production biologique de porcs en France : caractéristiques des élevages, impacts sur la santé, le bien-être et la qualité des produits
}

\author{
A. PRUNIER ${ }^{1,2}$, B. LEBRET ${ }^{1,2}$ \\ ${ }^{1}$ INRA, UMR1079 Systèmes d'Elevage Nutrition Animale et Humaine, F-35590 Saint-Gilles, France \\ 2 Agrocampus Ouest, UMR1079 Systèmes d'Elevage Nutrition Animale et Humaine, F-35000 Rennes, France \\ Courriel : Armelle.Prunier@rennes.inra.fr
}

\begin{abstract}
La production biologique de porcs en France est marginale par rapport à celle de porcs conventionnels : le cheptel de truies biologiques représente actuellement environ $0,5 \%$ de l'effectif total des truies reproductrices et le tonnage de viande produite correspond à environ $0,3 \%$ du tonnage de viande de porc (Agence Bio 2007). Cependant, le porc représente près du tiers du tonnage (en poids carcasse) des viandes biologiques produites en France. La production de porcs biologiques a beaucoup augmenté de 1999 à 2002 (figure 1), stagné jusqu'en 2006 puis montré une belle progression entre 2006 et $2007(+18 \%$ de truies et $+14 \%$ de viande). Comparativement aux autres pays européens, la France se situe en position inférieure pour la production de porcs biologiques, tant en valeur relative (environ $0,3 \%$ de porcs biologiques relativement au nombre
\end{abstract}

total de porcs produits en France contre $0,3 \%$ au Danemark, $0,4 \%$ en Allemagne, $0,6 \%$ en Grande-Bretagne et $0,8 \%$ en Suisse en 2006) qu'en valeur absolue (environ 5 fois moins de porcs biologiques produits en France qu'en Allemagne, 1,6 fois moins qu'au Danemark, 1,3 fois moins qu'en Grande-Bretagne en 2006) (Corepig). Cependant, avec les engagements pris lors du Grenelle de l'Environnement et compte tenu de l'intérêt des consommateurs pour les produits biologiques, on peut s'attendre à ce que la progression observée récemment en France se poursuive dans les années à venir.

Avant le 31 décembre 2008, la production de porcs biologiques des pays de l'Union Européenne (UE) était régie par deux règlements :

- le règlement $\mathrm{CE} \mathrm{n}^{\circ}$ 2092/91 du 24 juin 1991 qui définit des règles très

Figure 1. Evolution de la production de porcs biologiques en France depuis 1996 (agence Bio et Interbev).

Le tonnage de carcasses n'inclut pas la vente directe pour laquelle il n'y a pas de chiffres exacts mais qui représente probablement moins de $5 \%$ des viandes de porcs biologiques.

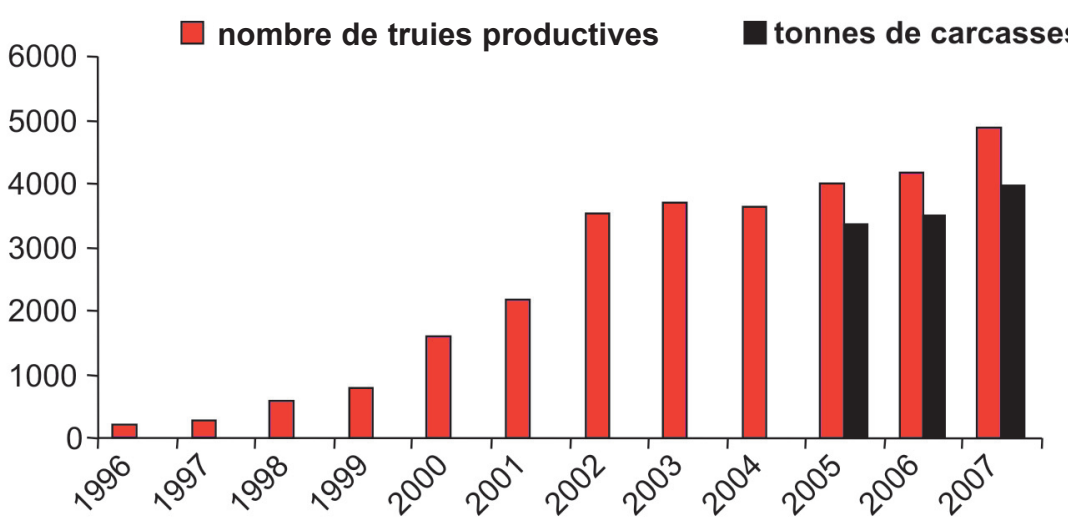

Année

générales sur la production biologique de produits agricoles,

- le règlement $\mathrm{CE} \mathrm{n}^{\circ} 1804 / 1999 \mathrm{du}$ 19 juillet 1999 qui définit de manière spécifique les règles qui s'appliquent aux productions animales biologiques dont le porc.

Les états membres de l'UE étaient alors autorisés à définir des règles nationales avec des normes de production plus strictes que celles définies dans la législation européenne. La France, la Belgique et le Danemark ont utilisé cette possibilité. En France, la production biologique de porcs était réglementée par le REPAB-F (Journal Officiel du 30 août 2000). Dans d'autres états membres de l'UE (par exemple la Grande-Bretagne, l'Allemagne et les Pays-Bas), des opérateurs privés ont développé leurs propres cahiers des charges.

Depuis le $1^{\text {er }}$ janvier 2009, de nouveaux règlements européens s'appliquent :

- le règlement $\mathrm{CE} \mathrm{n}^{\circ} 834 / 2007 \mathrm{du}$ 28 juin 2007 qui définit les objectifs et les principes généraux de la production et de l'étiquetage des produits biologiques,

- le règlement $\mathrm{CE} \mathrm{n}^{\circ} 889 / 2008 \mathrm{du}$ 5 septembre 2008 qui décrit les règles de production, d'étiquetage et de contrôle des produits biologiques.

Ces deux règlements sont applicables depuis le 1er janvier 2009 avec des délais d'application pouvant aller jusqu'au 31 décembre 2011 pour certaines mesures. Le règlement 834/2007, interdit l'établissement de règles nationales plus strictes que les règles européennes sauf si ces règles s'appliquent à l'ensemble de la production, y compris la 


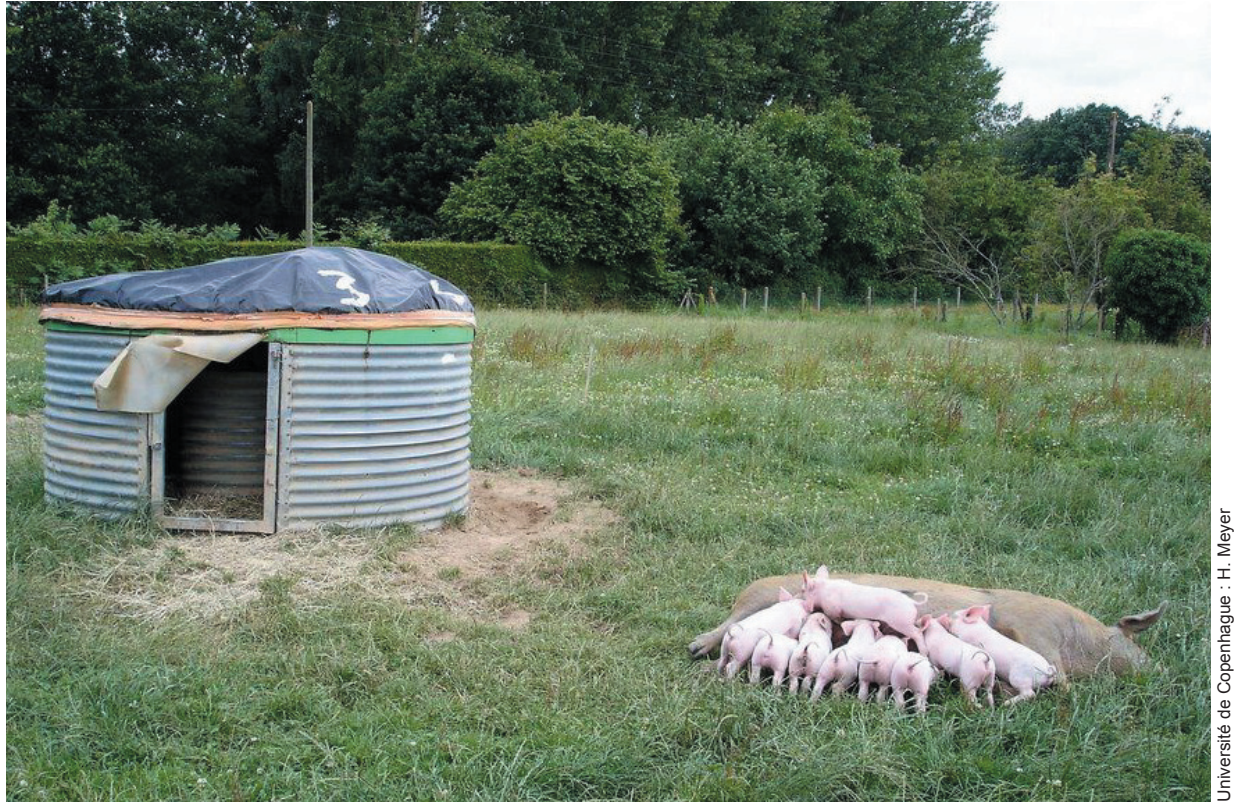

production conventionnelle. En pratique, cette disposition revient à interdire l'existence de règles nationales plus strictes que le socle européen pour la production biologique et conduit donc à la suppression du REPAB-F. L'existence de cahiers des charges privés reste possible dans la mesure où ils respectent les règles européennes.

A ces règlements, s'ajoutent les directives applicables à l'ensemble de la production porcine (directives 2001/88/CE du 23 octobre 2001 et 2001/93/CE du 9 novembre 2001) qui correspondent à des normes minimales en matière de logement, d'alimentation et de conduite d'élevage pour améliorer le bien-être des porcs. Cependant, ces directives n'apportent pas réellement de contraintes supplémentaires par rapport au règlement 889/2008.

D'une manière générale, les règles de la production biologique, qu'elles soient d'origine européenne, nationale ou privée, imposent des contraintes très fortes en matière de logement, de conduite et d'alimentation des porcs. Ces contraintes sont susceptibles d'avoir des répercussions sur les performances, le bien-être et la santé des animaux ainsi que sur la qualité des carcasses et des viandes. Dans cet article, nous décrirons d'abord brièvement les contraintes les plus importantes imposées par la législation, puis les caractéristiques des élevages de porcs biologiques en France et enfin les conséquences en termes de performances, bien-être et santé des animaux ainsi que de qualité des produits. Pour de nombreux paramètres, il n'existe pas d'expérimentation ni de résultats de terrain spécifiques à la production de porcs biologiques. Aussi, nous devrons également nous appuyer sur les commentaires d'experts qui suivent les élevages biologiques sur le terrain, sur les premiers résultats d'une enquête réalisée dans le cadre d'un projet européen (Corepig) ou encore sur des résultats d'élevages pratiquant des modes de production proches de ceux de l'élevage biologique des porcs.

\section{1 / Les principales contrain- tes législatives de la produc- tion de porcs biologiques en France}

Selon les termes mêmes de la législation (2092/91), «la production biologique est un système global de gestion agricole et de production alimentaire qui allie les meilleures pratiques environnementales [...], l'application de normes élevées en matière de bien-être animal et une méthode de production respectant la préférence de certains consommateurs à l'égard de produits obtenus grâce à des substances et à des procédés naturels». Il en résulte des contraintes fortes en termes d'alimentation, de logement et de traitements vétérinaires des animaux. Par ailleurs, le REPAB-F contrairement à l'ancienne réglementation européenne imposait une liaison forte de l'élevage au sol puisqu'au moins $40 \%$ de la matière sèche de la ration alimentaire devait être produite sur l'exploitation. La nouvelle réglementation européenne encourage une forte liaison des productions animales au sol, les effluents d'élevage devant être utilisés comme engrais pour les productions végétales : il s'agit d'inciter, et non d'obliger à respecter des valeurs seuils préétablies sur différents critères. L'épandage d'effluents excédentaires ne peut se faire que sur des exploitations ou entreprises respectant les règles de la production biologique. Enfin, le REPAB-F imposait une taille maximale des élevages de porcs biologiques : 1500 porcs charcutiers produits par an ou 200 truies présentes ou leur équivalent pour un élevage naisseur-engraisseur. Un dépassement du plafond était possible si $100 \%$ de l'alimentation des porcs était produite sur l'exploitation. La limite de taille des élevages a complètement disparu dans la nouvelle réglementation.

Le REPAB-F imposait que $90 \%$ de la matière sèche de la ration journalière des porcs soient d'origine biologique, contre $80 \%$ de la ration moyenne calculée sur l'année dans l'ancienne réglementation européenne. La nouvelle réglementation européenne impose que $90 \%$ de la ration des porcs soient d'origine biologique à partir du 1/01/2009 pour passer à $95 \%$ au $1 / 01 / 2010$ et à $100 \%$ au 1/01/2012 avec un calcul basé sur la moyenne annuelle. De plus, chaque ration journalière doit contenir au moins $75 \%$ d'aliment biologique. Le REPAB-F de même que la réglementation européenne précédente et actuelle (1804/1999 et 889/2008) imposent que les tourteaux soient extraits par pression, interdisent l'incorporation d'acides aminés de synthèse et d'Organismes Génétiquement Modifiés (OGM) même si une présence fortuite est tolérée dans la législation actuelle $(0,9 \%$ correspondant au seuil d'étiquetage). Ces contraintes rendent très difficile la formulation d'aliments avec une teneur équilibrée en acides aminés pour les porcs aux différents stades physiologiques (Sundrum et al 2007). Certaines matières premières biologiques d'origine animale (produits laitiers, produits et sous-produits du poisson, ovoproduits) ou minérale, de même que certains additifs nutritionnels (vitamines, oligoéléments) et technologiques (conservateurs) sont autorisés (listes positives). Des fourrages grossiers, frais, secs ou ensilés doivent être ajoutés à la ration journalière des porcs.

Concernant la conduite et le logement des animaux, le REPAB-F et la réglementation européenne ancienne et actuelle (1804/1999 et 889/2008) sont très proches. L'âge minimal au sevrage 
est de $40 \mathrm{j}$ pour les porcelets. La coupe de la queue et celle des dents ne doivent pas être effectuées même si des dérogations peuvent être accordées par l'autorité ou l'organisme de contrôle pour des raisons de santé ou de bien-être des animaux. La castration des porcs mâles est autorisée sans anesthésie jusqu'à $7 \mathrm{j}$ d'âge mais le nouveau règlement (889/2008) prévoit qu'elle soit effectuée sous anesthésie et/ou analgésie par du personnel qualifié avec un délai d'application de cette mesure expirant le 31 décembre 2011. Les conditions de logement doivent «garantir un niveau élevé de bien-être animal» et respecter les besoins comportementaux des animaux. En conséquence, les animaux doivent pouvoir se déplacer librement à tous les stades physiologiques et disposer d'espaces suffisants à l'intérieur et en plein air (figure 2). La gestion de la santé est axée sur la prévention des maladies en s'appuyant à la fois sur le choix des races, la conduite des animaux, et la qualité du logement et de l'alimentation (article 14 du règlement 834/2007). Les races doivent être choisies en fonction des capacités d'adaptation des animaux aux conditions locales, de leur vitalité et de leur résistance aux maladies. La préférence doit être donnée aux races et souches locales sans qu'il y ait d'obligation. Le REPAB-F imposait un âge minimal de 182 j d'âge à l'abattage pour les porcs charcutiers mais cette disposition n'existe pas dans le nouveau règlement européen.

L'utilisation préventive de médicaments allopathiques de synthèse est interdite de même que celle des substances destinées à stimuler la croissance ou à maîtriser la reproduction telles que celles destinées à induire la mise bas ou à synchroniser les chaleurs. Les produits phytothérapiques, les produits homéopathiques et certains additifs alimentaires (oligoéléments, vitamines synthétiques identiques aux vitamines naturelles...) énumérés dans les divers règlements (annexe II du 1804/1999,
REPAB-F puis annexe VI du 889/2008) sont autorisés. L'utilisation de médicaments vétérinaires allopathiques pour soigner des animaux malades ou blessés est autorisée, mais si le nombre de traitements est supérieur au seuil fixé par le règlement, l'animal est déclassé. En dehors des vaccinations, des traitements antiparasitaires et des plans d'éradication obligatoires, le nouveau règlement européen impose un nombre maximal de trois traitements sur une période de douze mois pour les animaux vivant plus d'un an (truies et verrats) et d'un seul traitement pendant leur vie pour les autres (porcs charcutiers). Le délai d'attente entre l'utilisation d'un médicament et l'abattage est doublé par rapport à l'élevage conventionnel. Les règles du REPAB-F étaient plus strictes puisqu'une limite existait également pour les traitements antiparasitaires ( 1 maximum pour les porcs charcutiers et 2 pour les truies ou verrats) et leur nombre devait être combiné avec celui des autres traitements

Figure 2. Superficies minimales par animal en production porcine biologique en $\mathrm{m}^{2}$.

Ces surfaces sont identiques dans les deux réglementations européennes successives (règlements CE n 1804/1999 et 889/2008) et dans l'ancienne réglementation française (REPAB F) concernant l'obligation de l'accès au plein air, des dérogrations sous certaines conditions sont possibles jusqu'au 21/12/2013.

A l'intérieur (superficie nette) A l'extérieur (aire d'exercice à l'exclusion des pâturages)
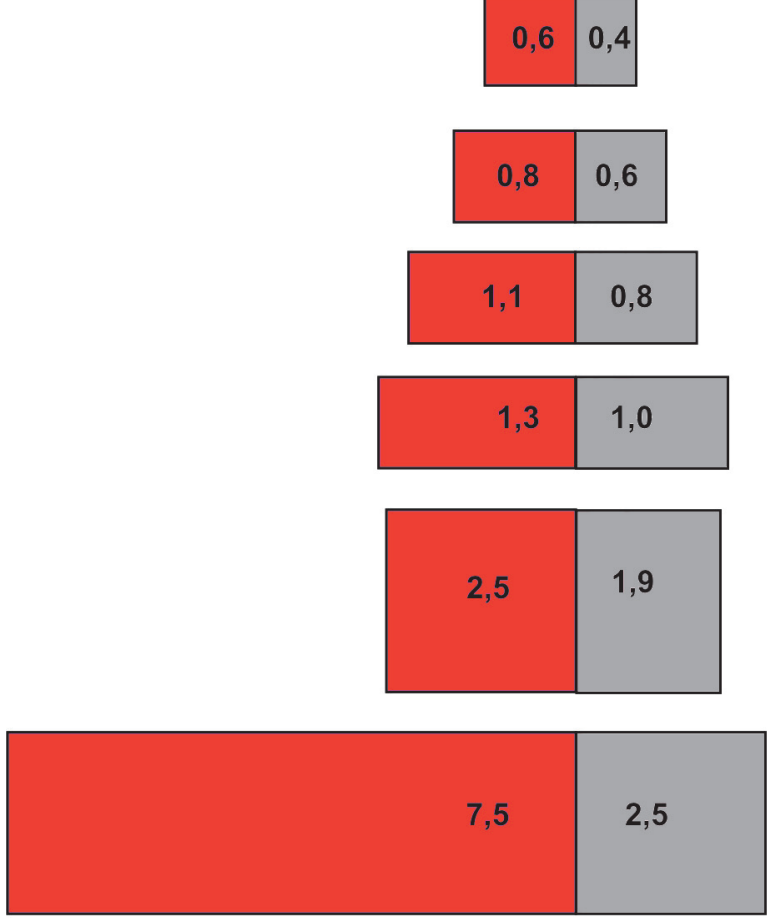

Porcelets

de plus de 40 jours et jusqu'à $30 \mathrm{~kg}$

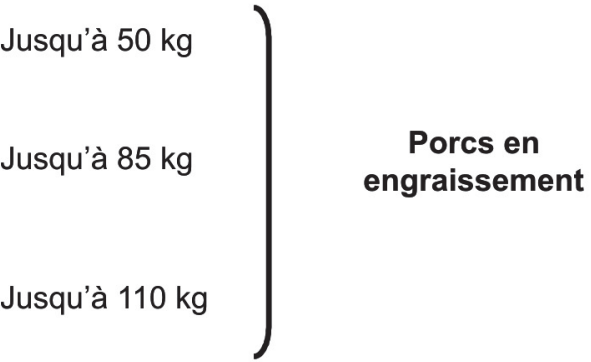

Femelles reproductrices

Truie allaitante

avec porcelets âgés de 40

jours au maximum 
allopathiques pour ne pas dépasser 3 au total (article 5.8.2).

Quel que soit le règlement, des mesures d'hygiène appropriées (nettoyage et désinfection) concernant les locaux et le matériel d'élevage doivent être prises pour prévenir le développement d'organismes pathogènes et les infections croisées entre animaux. Seuls les produits énumérés dans les annexes de la réglementation (annexe II du 1804/1999 et annexe VII du 889/2008) peuvent être utilisés. L'eau, la vapeur, la chaux et l'eau de javel font partie de ces produits.

\section{2 / Les principales caracté- ristiques de la production de porcs biologiques en France}

En 2007, le nombre total de truies biologiques présentes en France était d'environ 4900 , réparties dans 252 élevages avec une moyenne de 19,4 truies par élevage (Agence Bio 2007). Environ $20 \%$ de ces élevages comprenaient moins de 10 truies, $20 \%$ entre 10 et 20 truies et $60 \%$ plus de 20 truies. Toujours la même année, le nombre de porcs charcutiers produits était d'environ 41000 avec un poids moyen de la carcasse de $92 \mathrm{~kg}$ (Agence Bio 2007). Environ 3\% des élevages avaient moins de 10 porcs charcutiers, $9 \%$ entre 10 et 50 porcs et $88 \%$ plus de 50 porcs. Une partie de la production de porcs biologiques est en plein air avec $80 \%$ des truies gestantes et allaitantes, $70 \%$ des porcs en post-sevrage et seulement $5 \%$ des porcs charcutiers (Agence Bio 2007). Les principales régions productrices de porcs biologiques sont les Pays de la Loire (environ 30\% des truies en 2007), la Bretagne (environ $15 \%$ des truies), le Limousin et le Centre (environ 10\% des truies dans chaque région).

Chaque élevage biologique a des caractéristiques propres en termes de logement et de conduite, avec des variations au sein d'un même élevage pour un stade physiologique donné (données non publiées, enquête Corepig). Il est donc très difficile de décrire brièvement les traits principaux des élevages biologiques. Lorsque les animaux sont élevés en bâtiments, ces bâtiments sont généralement ouverts vers l'extérieur d'un côté et les animaux disposent toujours d'une litière de paille. Les truies gravides sont élevées en groupes, qu'elles soient en plein air ou en claustration. Les saillies sont très souvent naturelles notamment pour les élevages en plein air mais les éleveurs font également appel à l'insémination artificielle. Pour les élevages en plein air, il est fréquent de regrouper les truies dans des bâtiments semiouverts après le sevrage jusqu'à ce que la gestation soit confirmée. Pendant la période périnatale et la lactation, les truies sont le plus souvent logées individuellement mais certains éleveurs les regroupent au cours de la lactation. Les truies allaitantes sont toujours libres pendant la phase périnatale lorsqu'elles sont en plein air mais également dans certaines maternités en bâtiment. Cependant, dans certains élevages en bâtiments, les truies sont bloquées aux alentours de la mise bas jusqu'à $10 \mathrm{j}$, au plus tard, de lactation. La mise bas a lieu généralement sans surveillance des éleveurs mais la majorité réalisent des adoptions de porcelets dans les 2 j suivant la mise bas pour équilibrer les tailles de portée. Les génotypes utilisés sont variables mais le plus souvent de type conventionnel, le croisement Large White $x$ Landrace étant le plus fréquent côté maternel avec parfois des «gènes» Duroc; les croisements les plus fréquents côté paternel étant Large White X Piétrain, Piétrain pur (indemnes du gène de sensibilité à l'halothane) et Piétrain $x$ Duroc. Les races locales sont utilisées dans les petits élevages et il arrive que des croisements avec les races améliorées soient effectués.

Conformément au REPAB-F, au moins $40 \%$ de l'aliment des porcs est produit à la ferme et au moins $90 \%$ de l'aliment est d'origine biologique. Compte tenu de la nouvelle réglementation (cf. ci-dessus), ceci va probablement évoluer dans les années à venir. Dans la pratique, de très nombreux éleveurs produisent l'essentiel de l'ali- ment à la ferme ce qui permet de réduire les coûts de production des animaux. En effet, très peu d'aliments biologiques sont disponibles sur le marché et leur coût est élevé. Le sevrage des porcelets a lieu au plus tôt à $40 \mathrm{j}$ en conformité avec la réglementation, mais un sevrage plus tardif à 7 ou 8 semaines est pratiqué dans certains élevages (Corepig). La plupart des mâles sont castrés mais cette castration se fait souvent au-delà de $7 \mathrm{j}$ contrairement à ce qu'autorise la réglementation. Ceci s'explique en grande partie par le fait que les truies et les porcelets sont très souvent en liberté en plein air ce qui rend difficile la «capture» et la castration des porcelets. Le même problème se retrouve d'ailleurs dans les élevages conventionnels de plein air. Par ailleurs, la législation sur les pratiques de convenance (Directive 2001/93/EC) qui s'applique à tous les porcs indépendamment du système d'élevage semble mal appliquée au niveau européen (Fredriksen et al 2009). En conformité avec la réglementation, les dents et la queue ne sont pas coupées.

En termes sanitaires, les éleveurs de plein air pratiquent la rotation des prairies pour éviter les problèmes de parasitisme. La majorité des éleveurs utilisent des traitements contre les parasites internes des truies qu'elles soient en plein air ou en bâtiment et traitent les porcelets en post-sevrage, mais seulement une minorité d'éleveurs traitent les porcs charcutiers (Corepig). L'utilisation d'antibiotiques est très rare et la plupart des éleveurs pratiquent des vaccinations notamment contre le Rouget et le Parvovirus (Maupertuis et Calvar, communication personnelle). Selon les ingénieurs des Chambres d'Agriculture qui suivent ces élevages, les problèmes sanitaires

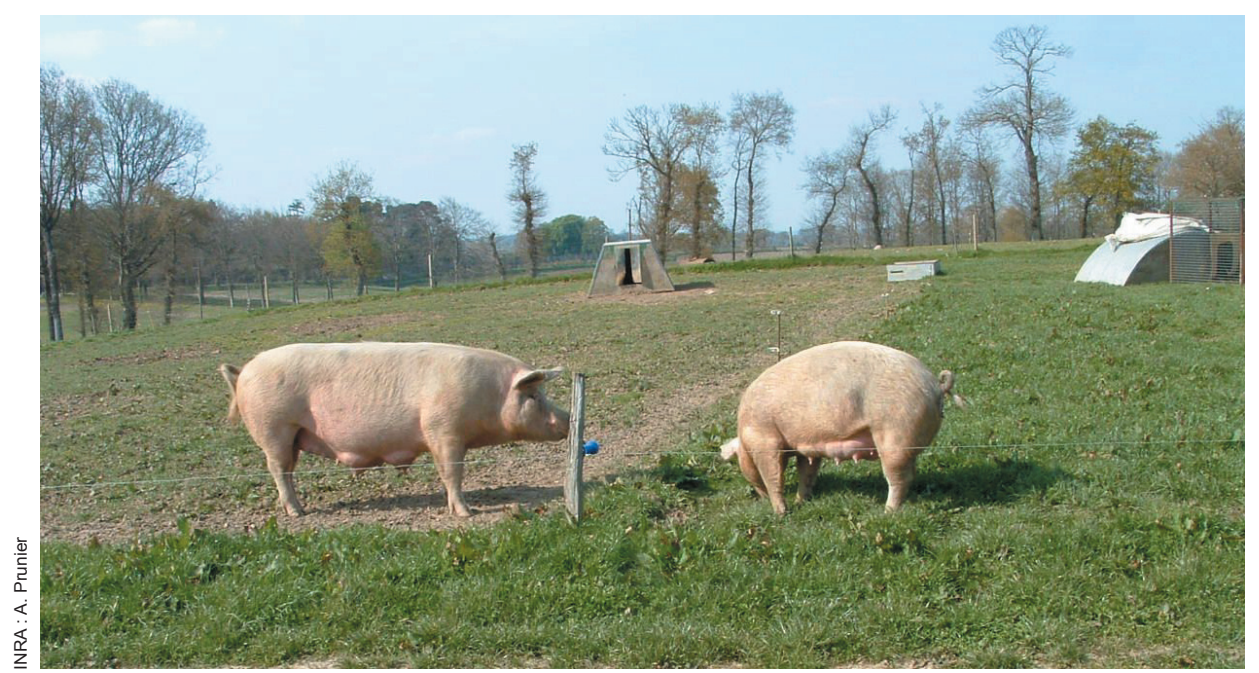


les plus fréquents sont la mortalité des porcelets sous la mère et les diarrhées des porcelets et, à moindre degré, les problèmes respiratoires chez les porcelets et les porcs charcutiers ainsi que les troubles de la fertilité et les boiteries chez les truies. De leur côté, les éleveurs considèrent également que la mortalité des porcelets sous la mère et les diarrhées en post-sevrage sont des problèmes importants (Corepig).

De très nombreux éleveurs de porcs biologiques utilisent des filières de vente courtes (vente directe, magasins coopératifs d'éleveurs), ce qui leur permet de mieux valoriser leurs produits. Les volumes de viande vendus directement par les producteurs aux consommateurs sont, par essence, très difficiles à estimer. A partir, d'une enquête réalisée par la Fédération Nationale d'Agriculture Biologique (FNAB) dans la région Centre, ces volumes auraient été de l'ordre d'une centaine de tonnes en 2005, ce qui représenterait moins de $5 \%$ des viandes porcines biologiques mais ces chiffres souffrent d'une grande incertitude. En 2007, environ la moitié de la viande de porcs biologiques, hors vente directe, a été vendue dans des Grandes et Moyennes Surfaces, $27 \%$ dans des magasins spécialisés pour produits biologiques et $18 \%$ dans des boucheries (commission Bio de l'Association Nationale Interprofessionnelle du Bétail et des Viandes Interbev).

\section{3 / Performances, bien-être et santé des porcs biolo- giques}

Il existe très peu de littérature scientifique concernant l'évaluation du bienêtre et de la santé dans les élevages de porcs biologiques. La plupart des publications européennes sont difficilement accessibles car publiées dans des revues techniques ou des rapports de congrès/journées d'études dans des langues autres que le français et l'anglais. Une étude est actuellement en cours sur une centaine d'élevages biologiques répartis dans six pays européens (Allemagne, Autriche, Danemark, France, Italie et Suède) pour quantifier et rechercher les facteurs de risques du parasitisme, de la mortalité des porcelets et des diarrhées en post-sevrage mais les résultats ne sont pas disponibles actuellement (Corepig).

Concernant le bien-être, la législation impose des normes en termes d'espace disponible par animal, de liberté de mouvement et d'enrichissement du milieu (litière, plein air) qui sont favorables à l'expression des divers comportements caractéristiques des porcs (par exemple nidification chez la truie, fouissage, bain de boue...). Des conséquences positives sur les lésions corporelles et la qualité des aplombs sont également prévisibles. Il a d'ailleurs été montré, chez des truies conventionnelles élevées en plein air dans des conditions de logement similaires à celles des élevages biologiques, que les animaux ont peu de lésions aux mamelles, peu de griffures sur le corps et de problèmes de boiterie (Vieuille et al 1996). De plus, la législation interdit l'épointage des dents, la coupe de la queue et imposera bientôt un traitement de la douleur lors de la castration des mâles, ce qui est également favorable au bienêtre des animaux. L'absence de coupe de queue ne pose pas de problème de cannibalisme, probablement parce que les porcs charcutiers sont élevés sur litière et disposent de beaucoup d'espace. Au total, les normes imposées par la réglementation sur l'élevage biologique sont favorables au bien-être des animaux (Spoolder 2007) même s'il est probable que des problèmes de bienêtre existent dans certains élevages. Ces problèmes peuvent être liés à l'alimentation (rationnement des truies gestantes, aliment déséquilibré en acides aminés), à des problèmes de logement/conduite (mortalité néonatale des porcelets) ou de santé (présence d'infections bactériennes ou virales, parasitisme interne ou externe).

Nous ne disposons pas de chiffres précis concernant les performances de reproduction des truies et la survie des porcelets dans les élevages biologiques. Les quelques chiffres disponibles en France concernent un très petit nombre d'élevages plein air de la région Pays de la Loire utilisant la Gestion Technique des Troupeaux de Truies (GTTT). Ces chiffres indiquent un nombre de porcs sevrés/truie productive/an de l'ordre de 19 et un nombre de porcs charcutiers produits/truie présente/an de l'ordre de 14 avec une mortalité (mort-nés compris) des porcelets jusqu'au sevrage de l'ordre de $30 \%$ et une mortalité du post-sevrage à l'abattage de l'ordre de 10\% (Maupertuis et Bordes 2007). Ces chiffres sont corroborés par les premiers résultats obtenus dans le projet Corepig. Une autre façon d'estimer la productivité des truies des élevages biologiques consiste à calculer le quotient entre le nombre de porcs charcutiers abattus et l'effectif de truies. Ce calcul réalisé sur les années 2006 et 2007 aboutit à environ 9 porcs produits/truie présente/an, ce qui est très faible au regard de la production conventionnelle (de l'ordre de 20 porcs en réalisant un calcul similaire utilisant les statistiques de 2007, Anonyme 2008). Cependant, il faut remarquer que le nombre de porcs biologiques abattus n'inclut pas les porcs commercialisés en vente directe qui représentent une part non négligeable de la production. Par ailleurs, les truies des très petits élevages (quelques truies) sont incluses. Ces truies sont le plus souvent de race locale peu prolifique et ont un rythme de reproduction très faible (souvent une portée par an). Elles représentent environ $15 \%$ de l'effectif total (Maupertuis communication personnelle) et abaissent fortement la productivité moyenne sans que l'on puisse dire exactement de combien.

Quoiqu'il en soit, la productivité relativement faible des truies en élevage biologique s'explique par de nombreuses raisons en sus de celles déjà évoquées pour les très petits élevages. Tout d'abord, une proportion élevée des élevages biologiques est en plein air, or la productivité des truies est plus faible en plein air qu'en bâtiment fermé (- 3 porcs sevrés/truie/an pour des élevages conventionnels de plein air par rapport à des bâtiments fermés, GTTT 2007) du fait d'un allongement de l'intervalle sevrage-saillie fécondante, d'une diminution du nombre total de porcelets nés et d'un accroissement de la mortalité des porcelets sous la mère. Une seconde raison, est que le rythme de reproduction est diminué en élevage biologique du fait de l'allongement de la lactation (au moins $40 \mathrm{j}$ pour toutes les truies mais souvent plus). Par ailleurs, l'allongement de la durée de la lactation et le fait que les truies soient regroupées pendant la lactation dans certains élevages favorisent probablement la reprise de la cyclicité pendant la lactation ce qui contribue à allonger l'intervalle sevrage-oestrus (Aumaître et Dagorn 1982, Rowlinson et Bryant 1982). Hulten et al (2006) ont d'ailleurs montré chez des truies conventionnelles logées en groupes et taries 7 semaines après la mise bas, que près de la moitié ovulent durant la lactation (près d'un quart avant 6 semaines) et ont un intervalle sevrage-oestrus très variable, compris entre 0 et $26 \mathrm{j}$ alors que pour les truies n'ayant pas ovulé en lactation, il est compris entre 2 et $8 \mathrm{j}$ avec un pic entre 4 et $6 \mathrm{j}$. Dans cette étude, la fréquence des oestrus de lactation augmente avec la parité des 
truies et varie avec la saison (plus fréquents en hiver et au printemps qu'en été et automne). Un autre point important expliquant la faible productivité des truies en élevage biologique est une mortalité des porcelets relativement élevée. Cette mortalité a lieu essentiellement pendant la mise bas et durant les premiers jours qui suivent la naissance avec comme raison principale l'écrasement (élevages plein air conventionnels : Berger et al 1997, Vieuille et al 2003 ; élevages biologiques : Feenstra 1999). Le froid, qui favorise le rapprochement entre les porcelets et la truie et diminue leur vitalité, est un facteur explicatif important de la mortalité relativement élevée des porcelets dans les élevages plein air, comme le suggèrent l'augmentation de la mortalité (Berger et al 1997) et la réduction de la température interne des porcelets (Guéguen et al 2000) pendant les mois d'hiver. Le même phénomène se rencontre probablement dans les élevages fermés lorsque l'environnement thermique est peu contrôlé. D'autres facteurs favorisent la mortalité des porcelets en plein air comme un enherbement insuffisant ou un mauvais drainage du sol (Berger et al 1997).

Concernant l'alimentation, les truies biologiques sont généralement rationnées pendant la gestation comme dans les élevages conventionnels, ce qui est connu pour favoriser l'expression de comportements stéréotypés ou l'existence de conflits entre truies lors de la distribution de l'aliment (MeunierSalaün et al 2007). L'accès des truies biologiques à des aliments riches en fibres permet probablement de réduire les problèmes, sans toutefois tous les régler. Ainsi, chez des truies conventionnelles élevées en plein air et pouvant pâturer, Vieuille et al (1996) ont montré un état d'engraissement insuffisant et des comportements stéréotypés fréquents chez certaines. Par ailleurs, compte tenu des difficultés à équilibrer correctement les rations alimentaires en acides aminés, des conséquences négatives sur la production laitière, la fonte des réserves corporelles des truies allaitantes et la croissance des porcelets aussi bien en lactation qu'après le sevrage, sont à attendre dans certains élevages biologiques.

Concernant la santé des animaux, l'élevage biologique présente des atouts mais aussi des inconvénients. La faible densité d'animaux, la moindre teneur en ammoniaque de l'air dans les bâtiments ainsi qu'un âge au sevrage tardif sont des facteurs favorables pour réduire la fréquence des maladies respiratoires et des diarrhées au sevrage. Ainsi, la fréquence des problèmes respiratoires détectés à l'abattoir (poumons avec signe de pneumonie et de pleurésie) est moins élevée dans les élevages biologiques que dans les élevages conventionnels (Hansson et al 2000, Wiskott 1998 cité par Baumgartner et al 2003). D'un autre côté l'accès au plein air, la présence de litière, la difficulté à conduire les animaux en bandes dans de petits élevages, le faible nombre de traitements allopathiques autorisés sont des facteurs défavorables pour le contrôle des pathogènes, pour plusieurs raisons. Ainsi, il est difficile dans les élevages biologiques de nettoyer et de désinfecter l'environnement des animaux ou d'empêcher le contact avec des animaux sauvages (sangliers, rongeurs, oiseaux...) vecteurs de maladies. Les quelques publications qui existent montrent que les parasites internes (essentiellement Oesophagostumum spp, Ascaris suum, Trichuris suis et Coccidia) et externes (essentiellement pou et gale) sont fréquents dans les élevages de porcs biologiques aussi bien en plein air (Danemark : Carstensen et al 2002) qu'en bâtiment (Autriche : Baumgartner et al 2003). Cependant, il est à noter qu'Oesophagostumum spp est aussi présent chez des truies conventionnelles quel que soit le logement, même si la fréquence est beaucoup moins élevée en bâtiment qu'en plein air (France : Lequeux 2006). De même, l'existence de taches blanches sur le foie qui signent la présence d'Ascaris peut être détectée en élevage conventionnel : une étude autrichienne a montré une fréquence similaire dans les deux systèmes (Wiskott 1998 cité par Baumgartner et al 2003) ; cette fréquence peut même être supérieure chez des porcs conventionnels, peut-être parce qu'ils sont plus jeunes et n'ont pas eu le temps de développer une immunité efficace (Hansson et al 2000). Le niveau des infections parasitaires reste souvent à un état subclinique dans les élevages de porcs biologiques, induisant seulement une réduction de l'efficacité alimentaire (Bonde et Sorensen 2004). La présence de salmonelles dans la viande à l'abattoir est un autre indicateur de la santé des animaux et est importante pour la qualité sanitaire des produits puisque les salmonelles sont transmissibles à l'homme. Une étude danoise a montré que la fréquence de salmonelles dans le jus de viande était inférieure chez les animaux issus d'élevage en plein air qu'ils soient biologiques ou conven- tionnels que chez ceux élevés en bâtiments (seulement conventionnels) mais le pourcentage d'animaux porteurs d'anticorps était supérieur pour l'élevage plein air, indiquant une contamination plus précoce (Bonde et Sorensen 2007). Les problèmes d'arthrite et d'arthrose semblent plus fréquents chez les porcs biologiques que chez les porcs conventionnels d'après une étude suédoise (Hansson et al 2000). Cependant cette même étude montre qu'au total, le pourcentage d'animaux avec un problème de santé est plus élevé en système conventionnel ( $28 \%$ des porcs) qu'en système biologique ( $17 \%$ des porcs) essentiellement du fait de la moindre fréquence d'abcès et de pleurésie.

\section{4 / Croissance des animaux et qualité de la viande des porcs biologiques}

Chez le porc, il est bien établi que, pour un génotype donné, le niveau alimentaire (restriction/à volonté) et la nature de la ration (rapport protéines/énergie, niveau et nature des acides aminés...) offertes aux porcs, leur mode de logement (type de logement, surface disponible par animal, aire d'exercice extérieure ou élevage en plein air favorisant l'activité physique) et les conditions climatiques (niveau moyen et amplitude des variations de températures) influencent la croissance relative des tissus maigres et gras et les propriétés de ces tissus et, par conséquent, la composition corporelle à l'abattage et la qualité des viandes (cf. synthèse de Lebret 2008). Les modalités d'alimentation comme de logement des porcs biologiques vont donc influencer les performances de croissance et les caractéristiques des produits (carcasses et viandes) qui en sont issus.

L'interdiction d'utiliser des acides aminés de synthèse implique que le besoin en protéines et en particulier en acides aminés essentiels soit couvert par l'incorporation dans la ration de matières premières biologiques riches en protéines. Cependant, celles-ci sont souvent produites en quantité insuffisante sur l'exploitation mais également peu disponibles sur le marché. La croissance des animaux, notamment le dépôt des tissus maigres peut donc être réduit faute d'apports suffisants en acides aminés essentiels. Ainsi, Sundrum et al (2000) ont comparé l'impact de quatre régimes alimentaires iso-énergétiques, supplémentés (conventionnel) ou non 
Tableau 1. Influence de la nature du régime alimentaire sur la vitesse de croissance (30 - $120 \mathrm{~kg}$ ) et la composition de la carcasse et du muscle Longissimus, d'après Sundrum et al (2000).

\begin{tabular}{|l|c|c|c|c|}
\hline Type de régime & $\begin{array}{c}\text { Conventionnel } \\
\text { C }\end{array}$ & $\begin{array}{c}\text { Biologique } \\
\text { B1 }\end{array}$ & $\begin{array}{c}\text { Biologique } \\
\text { B2 }\end{array}$ & $\begin{array}{c}\text { Biologique } \\
\text { B3 }\end{array}$ \\
\hline $\begin{array}{l}\text { Source de protéines } \\
\begin{array}{l}\text { Régime équilibré en } \\
\text { acides aminés }\end{array}\end{array}$ & $\begin{array}{c}\text { concentré de } \\
\text { protéines* }\end{array}$ & $\begin{array}{c}\text { fèverole }+ \\
\text { protéines de } \\
\text { pomme de terre } \\
\text { Oui }\end{array}$ & pois + lupin & $\begin{array}{c}\text { fèverole + } \\
\text { lupin }\end{array}$ \\
\hline $\begin{array}{l}\text { Vitesse de } \\
\text { croissance, g/j }\end{array}$ & $859 \pm 61 \mathrm{a}$ & $890 \pm 57 \mathrm{a}$ & $770 \pm 90 \mathrm{~b}$ & $766 \pm 67 \mathrm{~b}$ \\
$\begin{array}{l}\text { Taux de muscle, \% } \\
\text { Epaisseur de lard } \\
\text { dorsal, cm }\end{array}$ & $56,0 \pm 2,3 \mathrm{a}$ & $55,6 \pm 1,6 \mathrm{ab}$ & $54,3 \pm 2,0 \mathrm{bc}$ & $53,6 \pm 2,4 \mathrm{C}$ \\
$\begin{array}{l}\text { Teneur en lipides, } m . \\
\text { longissimus, \% }\end{array}$ & $2,4 \pm 0,3$ & $2,4 \pm 0,3$ & $2,4 \pm 0,3$ & $2,4 \pm 0,3$ \\
\hline
\end{tabular}

* farine de soja, farine de poisson, acides aminés synthétiques.

Pour chaque critère, les valeurs moyennes ( \pm écart-type) affectées d'une lettre différente sont différentes $(P<0,05)$

(biologique, trois compositions différentes) en acides aminés de synthèse sur les performances de croissance, la composition de la carcasse et les critères de qualité de la viande à l'abattage. Dans les trois régimes biologiques testés, les protéines étaient apportées soit par des fèveroles et des protéines de pomme de terre (B1) de façon à obtenir une composition en acides aminés comparable au régime conventionnel (C), des pois et du lupin (B2), ou des fèveroles et du lupin (B3), ces deux derniers régimes conduisant à des teneurs moyennes réduites en lysine $(-15 \%)$, méthionine + cystéine (- 20\%) thréonine (- 10\%) et tryptophane $(-13 \%)$ relativement aux régimes $\mathrm{C}$ et B1. Les aliments étaient distribués à volonté (croissance : 30 à $70 \mathrm{~kg}$ ) ou en quantité plafonnée $(2,60 \mathrm{~kg} / \mathrm{j})$ en finition $(70$ à $120 \mathrm{~kg})$. Comparativement au régime $\mathrm{C}$, le régime B1 ne modifiait pas les performances de croissance et la composition corporelle, alors que les régimes B2 et B3 induisaient une réduction de la vitesse de croissance et de la teneur en muscle des carcasses (tableau 1). Le type d'alimentation n'influençait pas l'épaisseur de lard dorsal, en revanche les régimes $\mathrm{B} 2$ et $\mathrm{B} 3$ conduisaient à une très forte augmentation de la teneur en lipides intramusculaires, composante favorable pour la qualité sensorielle (tendreté, jutosité) de la viande (Fernandez et al 1999a) relativement aux régimes B1 et C. Ainsi, la difficulté d'apporter une ration biologique équilibrée en acides aminés peut avoir des conséquences défavorables sur la croissance, notamment le développement du tissu musculaire et par suite la teneur en muscle, mais peut avoir des effets favorables sur le taux de lipides intramusculaires. L'augmentation de la teneur en lipides jusqu'à des valeurs comprises entre 2,5 et $3,5 \%$ peut se traduire par une amélioration de la jutosité et de la tendreté de la viande évaluée par un jury entraîné (Barton-Gade et Bejerholm 1985, Fernandez et al 1999a) ainsi qu'une amélioration de la texture, du goût et de l'acceptabilité globale de la viande par des consommateurs (Fernandez et al 1999b). Cependant, les résultats de Sundrum et al (2000) indiquent que la distribution des régimes B2 et B3 conduit à une augmentation de la teneur moyenne mais aussi de la variabilité individuelle en lipides intramusculaires avec des valeurs pouvant aller jusqu'à $4 \%$ (tableau 1). Or, une teneur en lipides de cet ordre peut être défavorable à l'acceptabilité de la viande par les consommateurs, en raison de la présence de gras visible (Fernandez et al 1999b).

Des fourrages grossiers doivent être ajoutés à la ration journalière soit directement, soit en offrant aux animaux l'accès à un parcours de plein air. Ces deux dispositions influencent fortement la vitesse et la composition du gain de poids (dépôts de protéines et de lipides) aux niveaux corporels et tissulaires, donc la composition de la carcasse et des tissus, ce qui peut se répercuter sur la qualité des viandes. La nécessité d'incorporer du fourrage à la ration des porcs biologiques peut conduire à apporter l'aliment «concentré» en quantité limitée (restriction) et distribuer le fourrage à volonté. D'un point de vue éthique, l'apport de fourrage permet de réduire l'impact négatif de la restriction alimentaire sur le bien-être des animaux qui ne pourraient sinon s'alimenter à satiété. D'un point de vue nutritionnel, la restriction alimentaire avec apport de fourrage à volonté, fait que les apports en énergie et protéines se trouvent alors souvent limités, avec pour conséquence une réduction de la vitesse de croissance des animaux et une modification des dépôts corporels et tissulaires. Cependant, l'impact de ces contraintes alimentaires sur la croissance des animaux et la qualité des produits dépend du mode d'élevage. Ainsi, Hansen et al (2006) ont montré que des porcs élevés en système d'élevage biologique (accès à une aire d'exercice extérieure) et recevant un aliment concentré biologique $(8,20 \mathrm{MJ} / \mathrm{kg}$ d'énergie nette, $20,9 \%$ de protéines brutes) à hauteur de $70 \%$ du besoin plus du fourrage à volonté (ensilage d'orge et de pois, ou ensilage d'herbe et de trèfle) avaient une croissance moins rapide et une teneur en muscle de la carcasse supérieure comparativement à des porcs élevés dans le même type de logement et alimentés à $100 \%$ avec l'aliment concentré biologique (tableau 2). Les porcs biologiques alimentés à volonté avaient eux mêmes une vitesse de croissance légèrement réduite mais une composition corporelle similaire à celle des porcs conventionnels (élevage en claustration, aliment conventionnel offert à volonté).

Ni la nature et le niveau de l'aliment alloué, ni le type d'habitat n'influencent le $\mathrm{pH}$ ultime, les pertes en eau (tableau 2), la couleur ou la teneur en pigments ou de la viande (non montrées). Cependant la teneur en lipides intramusculaires est réduite chez les 
Tableau 2. Effets de différents systèmes de production biologique des porcs sur la vitesse de croissance et la qualité des carcasses et de la viande, d'après Hansen et al (2006).

\begin{tabular}{|c|c|c|c|c|}
\hline Système d'élevage & Conventionnel & Biologique & Biologique & Biologique \\
\hline Type d'aliment concentré & Conventionnel & Biologique & Biologique & Biologique \\
\hline Niveau alimentaire & A volonté & A volonté & $70 \%$ à volonté & $70 \%$ à volonté \\
\hline Fourrage (à volonté) & - & - & Ensilage d'orge & Ensilage d'herbe \\
\hline Logement : accès extérieur & - & + & + & + \\
\hline Vitesse de croissance, $\mathrm{g} / \mathrm{j}$ & 999 a & $935 \mathrm{~b}$ & $726 \mathrm{c}$ & $731 \mathrm{c}$ \\
\hline Taux de muscle, $\%$ & 60,6 a & $60,4 \mathrm{a}$ & $61,3 b$ & $61,8 \mathrm{~b}$ \\
\hline Muscle Longissimus & & & & \\
\hline $\mathrm{pH} 24 \mathrm{~h}$ & 5,63 & 5,59 & 5,59 & 5,61 \\
\hline Pertes en eau, \% & 6,0 & 6,5 & 6,5 & 6,3 \\
\hline Teneur en lipides, $\%$ & $1,6 \mathrm{a}$ & $1,5 \mathrm{a}$ & $1,2 \mathrm{~b}$ & $1,2 \mathrm{~b}$ \\
\hline Tendreté (0 à 15) & $8,7 \mathrm{a}$ & 8,6 a & $7,5 \mathrm{~b}$ & $7,5 \mathrm{~b}$ \\
\hline Jutosité (0 à 15) & 8,9 & 8,8 & 8,7 & 8,8 \\
\hline
\end{tabular}

Pour chaque critère, les valeurs affectées d'une lettre différente sont différentes $(P<0,05)$.

animaux restreints avec complément de fourrage, quel que soit le type de fourrage, relativement aux animaux alimentés à volonté. La viande de ces animaux est jugée moins tendre que celle des porcs biologiques ou conventionnels alimentés à volonté, ceux-ci présentant une tendreté de viande équivalente. La réduction de la tendreté de viande des animaux restreints peut s'expliquer par leur teneur plus basse en lipides intramusculaires, ou un taux de renouvellement des protéines musculaires et un potentiel protéolytique post mortem plus faibles suite à leur vitesse de croissance réduite par rapport aux animaux alimentés à volonté. Ainsi, les différences de qualité de viande ne sont pas associées directement au système de production (biologique vs conventionnel), mais aux conséquences de certaines conduites d'élevage (restriction alimentaire) appliquées en système biologique dans cette étude. Les travaux de Oksbjerg et al (2005) combinant différentes conditions de logement (intérieur avec courette extérieure ou plein air sur parcours) et différents niveaux alimentaires (à volonté ou restriction de $20 \%$ ) conduisaient aux mêmes conclusions. Ces effets de la restriction alimentaire sur les performances, la composition corporelle et la qualité de viande sont conformes aux nombreuses données de la littérature indiquant que la restriction chez le porc en croissance affecte plus fortement le dépôt de lipides que celui des protéines au niveau corporel mais aussi au niveau intramusculaire, conduisant à la production de carcasses plus maigres et de viandes moins riches en lipides. Par suite, la tendreté ou la jutosité de la viande peuvent être altérées (Affentranger et al 1996, Blanchard et al 1999, Lebret 2008).
L'impact du système de production biologique sur la croissance et la qualité de la viande peut également provenir du mode de logement des animaux. Millet et al (2005) ont évalué l'influence de l'alimentation : 100\% biologique ou $100 \%$ conventionnelle incluant des acides aminés de synthèse (lysine, thréonine, méthionine), chez des porcs élevés dans deux types d'habitat : «biologique» (bâtiment sur litière de paille avec courette extérieure, $4 \mathrm{~m}^{2} /$ porc au total) ou conventionnel (claustration, sol plein avec une partie caillebotis, $1 \mathrm{~m}^{2} /$ porc). Ces auteurs ont montré un effet marqué des conditions de logement sur les caractéristiques zootechniques, avec une augmentation de la consommation alimentaire, de la vitesse de croissance et de l'épaisseur de lard dorsal et une moindre teneur en muscle des carcasses des porcs élevés en logement biologique relativement au logement conventionnel, alors que le type d'alimentation (biologique ou conventionnelle) ne modifiait pas ces paramètres. Aucune différence n'était observée sur les critères de qualité de viande : vitesse et amplitude de chute $\mathrm{du} \mathrm{pH}$, pertes en eau ou couleur de la viande, selon le type d'habitat ou le type d'alimentation (Millet et al 2005).

Concernant les conditions de logement, des systèmes extensifs en parcs sont également rencontrés en production porcine biologique. Olsson et al (2003) ont comparé des porcs de génotype (Landrace $x$ Yorkshire) $x$ Hampshire dont la moitié porteurs et l'autre moitié non porteurs de l'allèle $R N^{-}$(allèle défavorable du gène $R N$ à effet majeur sur la qualité, toujours en ségrégation dans les populations porcines suédoises), élevés en système biologique ou conventionnel. En système biologique, les animaux étaient élevés en parc (groupe de 40 animaux, $6000 \mathrm{~m}^{2}$ ) et recevaient un aliment biologique $(12,6 \mathrm{MJ} / \mathrm{kg}$ d'énergie métabolisable, $15 \%$ de protéines brutes) ; en système conventionnel, les animaux étaient élevés en claustration (cases de 8 porcs) et recevaient un aliment conventionnel $(12 \mathrm{MJ} / \mathrm{kg}$ d'énergie métabolisable, $16,8 \%$ de protéines brutes, supplémenté en lysine, méthionine et thréonine). La vitesse de croissance et l'épaisseur de lard dorsal étaient plus élevées et le taux de muscle inférieur chez les porcs biologiques, probablement en raison de la teneur en énergie plus élevée du régime biologique, et ce malgré un besoin énergétique vraisemblablement supérieur pour ces animaux élevés en plein air. L'élevage biologique entraînait une légère dégradation de la qualité technologique ( $\mathrm{pH}$ ultime plus bas, pertes en eau accrues) et sensorielle (force de cisaillement plus élevée, moindre jutosité) de la viande des porcs non porteurs de l'allèle $R N^{-}$ $(\mathrm{rn}+/ \mathrm{rn}+)$ comparativement aux non porteurs élevés en système conventionnel, ou aux porcs $\mathrm{RN}^{-} / \mathrm{rn}+$ élevés en système biologique ou conventionnel (Jonsäll et al 2002, Olsson et al 2003). Ainsi, l'effet du système de production sur la qualité de viande dépendait du génotype au locus $R N$. Pour des animaux non porteurs, comme c'est le cas en France, le système biologique considéré dans cette étude tendait à altérer la qualité de la viande. Pour les porcs conventionnels, une dégradation de la qualité de viande de porcs élevés en plein air relativement à l'élevage en claustration est parfois rapportée $(\mathrm{pH}$ ultime plus bas en relation avec une teneur en glycogène musculaire plus élevée) alors que d'autres études ne montrent pas de différence, voire une amélioration de la qualité (tendreté) (Lebret 2008). Ceci met en évidence la 
plus grande variabilité de qualité de la viande engendrée par les systèmes d'élevages plein air, qu'ils soient conventionnels ou biologiques, comparativement aux systèmes en claustration.

Pour résumer, la conduite alimentaire : niveau alimentaire (restriction éventuelle) et nature du régime (sources de protéines biologiques conditionnant les apports en acides aminés) ainsi que les conditions de logement (intérieur avec courette extérieure ; plein air sur parcours...) des porcs élevés en systèmes biologiques peuvent avoir des conséquences variées, parfois opposées, sur les performances de croissance, la composition de la carcasse et les caractéristiques tissulaires. La qualité des viandes de porc issues des élevages biologiques peut donc présenter une certaine variabilité, qui résulte de la diversité des systèmes de production rencontrés.

\section{Conclusion}

La réglementation sur l'élevage de porcs biologiques impose des normes sur le logement, l'alimentation et la conduite des porcs. Cependant, ce cahier des charges laisse des marges de manœuvre importantes aux éleveurs si bien qu'il n'y a pas de mode de production biologique des porcs précis et homogène. Ceci engendre une grande diversité de situations, conduisant très probablement à des différences en termes de performances, de bien-être et de santé des animaux ainsi que de qualité des produits qui en sont issus. Mieux connaître la diversité des élevages et les liens entre logement, alimentation, conduite et réponses biologiques des animaux contribuerait à améliorer les élevages sur différentes dimensions de la production : performances zootechniques, bien-être et santé des animaux, qualité des produits. La rentabilité économique, de même que la qualité du travail, dimensions non traitées dans cet article, sont également essentielles à prendre en compte afin d'identifier des pistes permettant in fine de développer cette production. Cependant, l'acquisition de connaissances ne suffira pas les éleveurs doivent pouvoir bénéficier d'encadrements techniques, disposer d'aliments biologiques en quantité et qualité suffisantes à prix raisonnable, notamment pour les porcelets, d'animaux reproducteurs adaptés à leur besoin... pour que la production porcine biologique puisse se développer. C'est donc toute une filière qui est concernée.

\section{Remerciements}

Les auteurs tiennent à remercier C. Calvar (Chambre d'Agriculture de Bretagne de Vannes) et F. Maupertuis (Chambre d'Agriculture des Pays de la Loire, d'Ancenis) pour leur lecture critique du manuscrit et leurs conseils.

\section{Références}

Affentranger P., Gerwig C., Seewer G.J.F., Schwörer D., Künzi N., 1996. Growth and carcass characteristics as well as meat and fat quality of three types of pigs under different feeding regimens. Livest. Prod. Sci., 45, 187-196.

Agence Bio, 2007. L'agriculture biologique française en 2007. http://www.agencebio.org/ upload/p.agesEdito/fichiers/production-BioFrance2007.pdf.

Anonyme, 2008. Les élevages suivis en GTTT (Gestion Technique des Troupeaux de Truies). Résultats GTTT des élevages en plein air, 37 ; Composition du cheptel français. Résultats GTTT des élevages en plein air, 8 ; L'abattage industriel des porcs. Le Porc par les chiffres, Editions ITP, Paris, France, 52p.

Aumaître A., Dagorn J., 1982. Influence de la durée de lactation sur la fécondité et la prolificité de la truie. Ann. Zootech., 31, 431-444.

Barton-Gade P.A., Bejerholm C., 1985. Eating quality of pork. Pig Farming, 33, 56-57.

Baumgartner J., Leeb T., Gruber T., Tiefenbacher R., 2003. Husbandry and animal health on organic pig farms in Austria. Anim. Welfare, 12, 631-635.

Berger F., Dagorn J., Le Denmat M., Quillien J.P., Vaudelet J.C., Signoret J.P., 1997. Perinatal losses in outdoor pig breeding. A survey of factors influencing piglet mortality. Ann. Zootech., 46, 321-329.

Blanchard P.J., Ellis M., Warkup C.C., Hardy B., Chadwick J.P., Deans G.A., 1999. The influence of rate of lean and fat tissue development on pork eating quality. Anim. Sci., 68, 477-485.

Bonde M., Sorensen J.T., 2004. Herd health management in organic pig production using a quality assurance system based on hazard analysis and critical control points. NJASWageningen. J. Life Sci., 52, 133-143.
Bonde M., Sorensen J.T., 2007. Effect of pig production system and transport on the potential pathogen transfer risk into the food chain from Salmonella shed in pig faeces. Proc. $3^{\text {rd }}$ Int. Cong. Eur. Integrated Project Quality Low Input Food, Université de Hohenheim, Allemagne, 2023 mars 2007, 104-107.

Carstensen L., Vaarst M., Roepstorff A., 2002. Helminth infections in Danish organic swine herds. Vet. Parasitol., 106, 253-264.

Corepig, Prevention of selected diseases and parasites in organic pig herds - by means of a HACCP based management and surveillance programme. http://corepig.coreportal. org/?page $\mathrm{id}=1$.

Directive 2001/93/CE, 2001. Commission du 9 novembre 2001 établissant les normes minimales relatives à la protection des porcs. J. Off. Comm. Eur., 1.12.2001.

Feenstra A., 1999. A health monitoring study in organic pig herds. Proc. N.J.F. Seminar, Horsens, Danemark, 16-17 september, 107-112.

Fernandez X., Monin G., Talmant A., Mourot J., Lebret B., 1999a. Influence of intramuscular fat content on the quality of pig meat - 1 . Composition of the lipid fraction and sensory characteristics of $m$. longissimus lumborum. Meat Sci., 53, 59-65.

Fernandez X., Monin G., Talmant A., Mourot J., Lebret B., 1999b. Influence of intramuscular fat content on the quality of pig meat - 2 . Consumer acceptability of $\mathrm{m}$. Longissimus lumborum. Meat Sci., 53, 67-72.

Fredriksen B., Font i Furnols M., Lundström K., Migdal W., Prunier A., Tuyttens F., Bonneau M., 2009. Practice on castration of piglets in Europe, Animal, sous presse.

FNAB, 2006. Estimation des volumes vendus en direct par les producteurs aux consommateurs. GRAB-1 ere estimation RARE-CT-09-2006.
Guéguen R., Quillien J.P., Meunier-Salaün M.C., Salaün C., Callarec J., 2000. Incidence du système de logement et de la saison sur la survie néonatale des porcelets. Journ. Rech. Porcine Fr., 32, 123-128.

Hansen L.L., Claudi-Magnussen C., Jensen S.K., Andersen H.J., 2006. Effect of organic pig production systems on performance and meat quality. Meat Sci., 74, 605-615.

Hansson I., Hamilton C., Ekman T., Forslund K., 2000. Carcass quality in certified organic production compared with conventional livestock production. J. Vet. Med., Series B 47, 111-120.

Hulten F., Wallenbeck A., Rydhmer L., 2006. Ovarian activity and oestrous signs among group-housed, lactating sows: influence of behaviour, environment and production. Reprod. Dom. Anim., 41, 448-454.

INTERBEV, Commission Bio. Les viandes biologiques : naturalité et authenticité commission Bio. http://www.interbev.fr/fileadmin/ docs/dossier-presse-viande-bio.pdf.

Jonsäll A., Johansson L., Lundström K., Andresson K.H., Nilsen A.N., Rivsik E., 2002. Effects of genotype and rearing system on sensory characteristics and preference for pork $(M$ Longissimus dorsi). Food Quality Preference, $13,73-80$.

Lebret B., 2008. Effects of feeding and rearing systems on growth, carcass composition and meat quality in pigs. Animal, 2, 1548-1558.

Lequeux G., 2006. L'infestation des truies par Oesophagostomum spp. : Enquête en abattoir et en élevages dans 1'ouest de la France. Thèse, Ecole Vétérinaire de Nantes, 100p.

Maupertuis F., Bordes A., 2007. Les références technico-économiques sont issues du réseau porc biologique des Pays de la Loire de 2003 à 2006. Chambre d'Agriculture Pays de la Loire, $2 \mathrm{p}$. 
Meunier-Salaün M.C., Bizeray D., Colson V., Courboulay V., Lensik J., Prunier A., Rémience V., Vendenheede M., 2007. Bien-être et élevage des porcs. INRA Prod. Anim., 20, 73-80.

Millet S., Raes K., Van den Broeck W., De Smet S., Janssens G.P.J., 2005. Performance and meat quality of organically versus conventionally fed and housed pigs from weaning till slaughtering. Meat Sci., 69, 335-341.

Oksbjerg N., Strudsholm K., Lindahl G., Hermansen, J.E., 2005. Meat quality of fully or partly outdoor reared pigs in organic production. Acta Agric. Scand. Section A., 55, 106-112.

Olsson V., Andersson K., Hansson I., Lundström K., 2003. Differences in meat quality between organically and conventionally produced pigs. Meat Sci., 64, 287-297.

Règlement CEE n 2092/91, 1991.Conseil du 24 juin 1991 concernant le mode de production biologique de produits agricoles et sa présentation sur les produits agricoles et les denrées alimentaires. J. Officiel Comm. Eur., 22.7.1991.

Règlement $\mathrm{CE} \mathrm{n}^{\circ} 889 / 2008$, 2008. Commission du 5 septembre 2008 portant modalités d'ap- plication du règlement $(\mathrm{CE}) \mathrm{n}^{\circ} 834 / 2007 \mathrm{du}$ Conseil relatif à la production biologique et à l'étiquetage des produits biologiques en ce qui concerne la production biologique, l'étiquetage et les contrôles. J. Officiel Comm. Eur., 18.9.2008.

Règlement CE n 1804/1999, 1999. Conseil du 19 juillet 1999 modifiant, pour y inclure les productions animales, le règlement $\mathrm{CEE}$ $n^{\circ} 2092 / 91$ concernant le mode de production biologique de produits agricoles et sa présentation sur les produits agricoles et les denrées alimentaires. J. Officiel Comm Eur., 24.8.1999.

REPAB-F, 2000. Cahier des charges concernant le mode de production et de préparation biologique des animaux et des produits animaux définissant les modalités d'application du règlement CEE n 2092/91 modifié du Conseil et/ou complétant les dispositions du règlement CEE $n^{\circ}$ 2092/91 modifié du Conseil. J. Off. République française du 30 août 2000 .

Rowlinson P., Bryant M.J., 1982. Lactational oestrus in the sow. 2. The influence of grouphousing, boar presence and feeding level upon the occurrence of oestrus in lactating sows. Anim. Prod., 34, 283-290.

Spoolder H., 2007. Perspective animal welfare in organic farming system. J. Sci. Food Agric., 87, 2741-2746.

Sundrum A., Büftering L., Henning M., Hoppenbrock K.H., 2000. Effects of on-farm diets for organic pig production on performance and carcass quality. J. Anim. Sci., 78, 1199-1205.

Sundrum A., Nicholas P., Padel S., 2007. Organic farming: challenges for farmers and feed suppliers. In: Recent advances in Animal Nutrition. Garnsworthy P.C., Wiseman J. (Ed), Nottingham University Press, 239-260.

Vieuille C., Cariolet R., Madec F., MeunierSalaün M.C., Vaudelet J.C., Signoret J.P., 1996. Evaluation du bien-être en élevage chez la truie gestante. Approche comparative dans quatre systèmes de logement. Journ. Rech. Porcine Fr., 28 307-318.

Vieuille C., Berger F., Le Pape G., Bellanger D., 2003. Sow behaviour involved in the crushing of piglets in outdoor farrowing huts - a brief report. Appl. Anim. Behav. Sci., 80, 109-115.

\section{Résumé}

La production de porcs biologiques en France est marginale par rapport à celle des porcs conventionnels (moins de $1 \%$ en tonnage) mais en progression constante. La production porcine biologique est fortement encadrée par la réglementation qui impose des contraintes fortes en termes de logement, d'alimentation et de conduite d'élevage. Ces contraintes peuvent influencer les performances, la santé et le bien-être des animaux ainsi que la qualité des carcasses et des viandes. Les données existantes suggèrent une grande diversité des types d'élevages de porcs biologiques et in fine des conséquences du mode de production biologique sur le bien-être et la santé des animaux vivants ainsi que sur la qualité des produits. D'une façon générale, le mode de production biologique offre des atouts pour répondre à l'attente des consommateurs concernant le bien-être animal et la qualité des produits, mais les éleveurs doivent être vigilants en matière de santé des animaux, d'équilibre des rations alimentaires, surtout pour les porcelets et les truies allaitantes, et de qualité des carcasses.

\section{Abstract}

\section{Organic pig production in France: characteristics of farms, impacts on health and welfare of animals and on product quality}

The importance of organic pig production is very low in France compared to that of conventional pigs (less than $1 \%$ of pork production) but increases regularly. Organic pig production is organised by the legislation that imposes numerous constraints in terms of housing, feeding and husbandry. These constraints are likely to influence performances, health and welfare of the pigs as well as the quality of carcasses and meat. Existing knowledge suggests a high diversity in the types of organic pig farms and hence on the consequences of the organic farming on pig welfare and health as well as on the quality of the products. Overall, organic farming offers good opportunities to respond to consumers' wishes regarding animal welfare and quality of food products provided that pig producers pay attention to animal health, nutritional balance of the diets, especially for piglets and lactating sows, and quality of carcasses.

PRUNIER A., LEBRET B., 2009. La production biologique de porcs en France : caractéristiques des élevages, impacts sur la santé, le bien-être et la qualité des produits. Inra Prod. Anim., 22, 179-188.
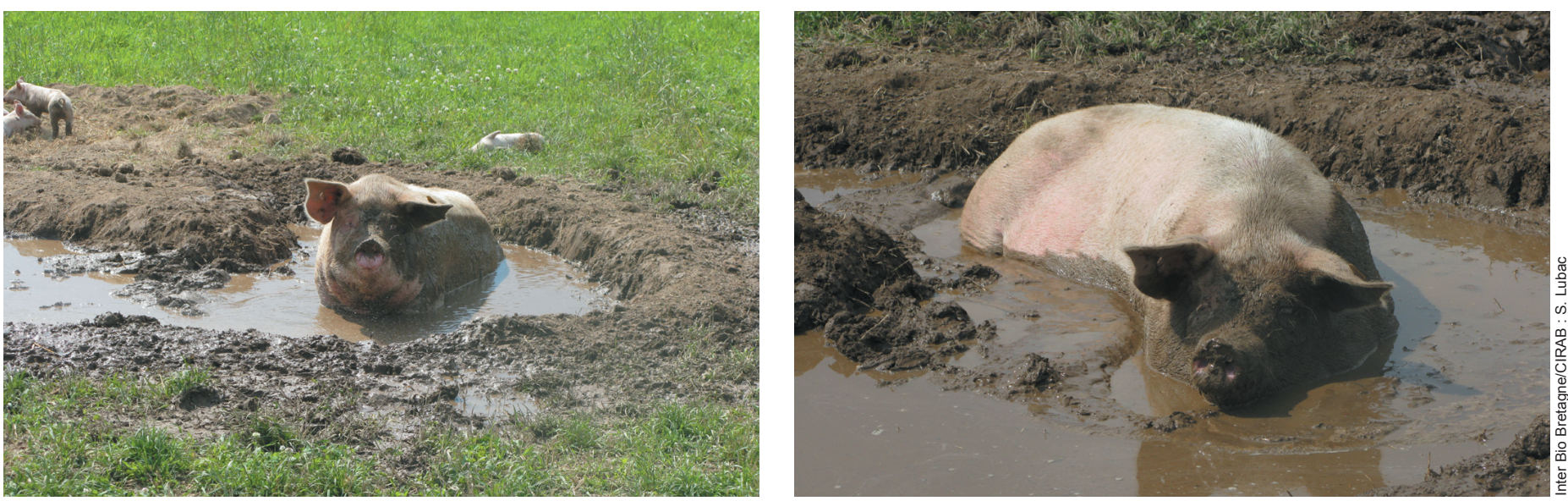